\title{
Developing Web Browser-Jan
}

\author{
Rajeswari. $\mathrm{M}^{1}$, Brindha. $\mathrm{S}^{2}$, Sindhuja. $\mathrm{N}^{3}$, Jaganathan. $\mathrm{S}^{4}$ \\ ${ }^{I}$ (Assistant professor, cse, Angel college of Engineering and technology, Chennai, India) \\ ${ }^{2}$ (Student, cse, Angel college of Engineering and technology, Chennai, India) \\ ${ }^{3}$ (Student, cse, Angel college of Engineering and technology, Chennai, India) \\ ${ }^{4}$ (Student, cse, Angel college of Engineering and technology, Chennai, India)
}

\begin{abstract}
A web browser is a software application for retrieving, presenting and traversing information resources. It can also be defined as an application software or program designed to enable users to access, retrieve and view documents and other resources on the Internet. The major web browsers are Chrome, Firefox, Internet Explorer, Opera, and Safari. JAN web browser mainly focused for users who need efficient resource and save their time. Our web browser is based on the concept of data mining where the data is extracted from the huge resource called internet. JAN browser consists of additional feature like virtual keyboard which is used for security purpose where the password cannot be hacked and also our browser support HTTP Secure and offer quick and easy ways to delete the web cache, cookies, and browsing history, it also shown the current time and date using time and date the user can set the alarm for their work. This browser also has a speed dialing concept where the speed dialing is assigned in network wise whereas in existing browser this type of concept is not found. And also this browser will give the user to save their document in their required format. So our project will result in the satisfaction of users for what they expect
\end{abstract}

Keywords - Browser, Speed dialing, Secure, virtual keyboard

\section{INTRODUCTION}

The current world is running faster in all environments the user doesn't have time to wait for a moment to get any resources. The present browser made the user to search a resource in difficult manner for example in present browser the user need to memorize the website and URL to search correct location else they need to view all the website which was given by the browser. Browser doesn't have any security for internet usage anyone can access other's browser without any authentication. To overcome above given difficulties a new web browser is developed which help the user to get their resources efficiently and provide security to browser. This project contain features such as speed dialing which provide all website which were frequently used by the user and the website are separate depending upon their fields. For example audio and video speed dialing in this we provide all website which is related to the field it make the user to get their required website easily and user don't need of memorize. Similarly this project contains all website which is related to their fields. Our web browser contains the login page where every new user should register for getting their username and password to login our browser. It handles a new method called Virtual Keyboard which is not like a normal keyboard. Its special feature is shuffling the keys each time. To avoid hacking this keyboard is used.

\section{Existing System}

The browsers are playing a vital role in retrieving and traversing information resources. So those browsers should be well effective for the users to use. Most of the web browser's does not have login page for the security purpose. In the current browsers there is no security for the internet usage, any user can use others PC browser without any authentication.

Major problem in the recent network world is hacking. Here we are to going concentrate on password hacking. The password hacking is affecting the personal details through Email, Face book, etc.

And moreover web browser does not include speed dialing for the network wise. The current web browsers make the user to waste their valuable time by either memorizing the required website or they want to view all the result websites provided by the browser. We ensure that the above drawbacks will be fulfilled by our project.

\section{II.1 Drawback}

The browser has many drawbacks in Security, Searching Resource .In this modern world, browser play an important role in sharing resources. So that kind of resources should be securable. And also the speed dial concepts are not included for separate network like education, audio and video network. Because of these methods the user's valuable time is wasted. So the better way to solve all these drawbacks is by our Jan browser which processes all this in a smarter and securable manner. 


\section{Proposed System}

The problems which we identified from the existing browsers will be solved by implementing a new browser which provides security, efficient internet usage and resource finding. We concentrate on above features by developing a web browser called Jan.

In our browser, security is provided in two ways. First is by securing the password which includes virtual keyboard. In virtual keyboard, whenever the key is pressed the content of key is not stored in the buffer and also key is shuffled each time. But in the normal keyboard the content is stored and by using the key locker software the third person can easily hack the password which could not be done by virtual keyboard so the personal information's will be saved. PC browser.

Latter is by securing the internet usage through login for the user. It avoids unwanted users to access

Then also our browser has the speed dialing tab for the education, audio and video network. We will provide all the necessary websites in the network, the user can choose the respective site which they want to access. These are the important feature in our paper which will help all users to use them in an intractable manner.

\section{Technical Feasibility And Discussions}

Every new project we start to develop should be analyzed thoroughly about its technical possibilities to implement, while designing itself. Because we can propose a system with some new features than the existing systems. So there may be lot of impossibilities with our new concepts in practical. So analyzing the technical feasibility gives us the confidence to make next steps in our project. We have proposed the system with front end as .NET and the back end is SQL. The querying the database is very effective and the connectivity between SQL and .NET are easier in nature.

\section{Experimental Results And Discussions}

Below figs are shown the function of JAN Browser. Home Page is shown in fig 1 default it set as JAN browser

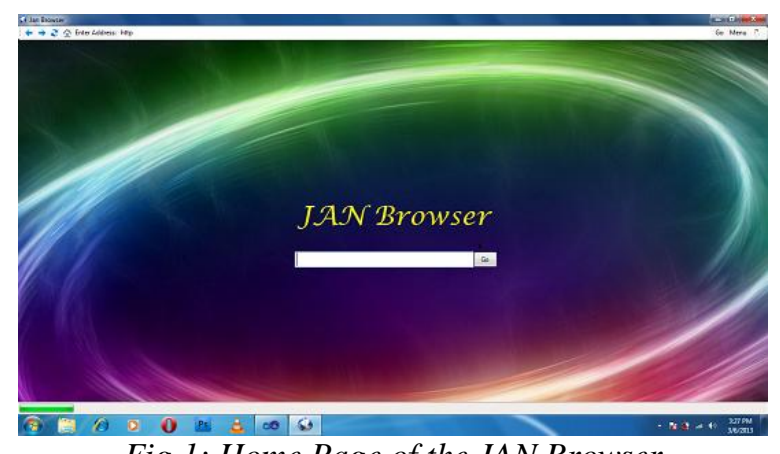

Fig 1: Home Page of the JAN Browser

In home the user can search required webpage through keywords or type the URL in URL text field print, exit.

In fig 2 shown the File Menu in JAN browser the File Menu options are new tab, new window, save,

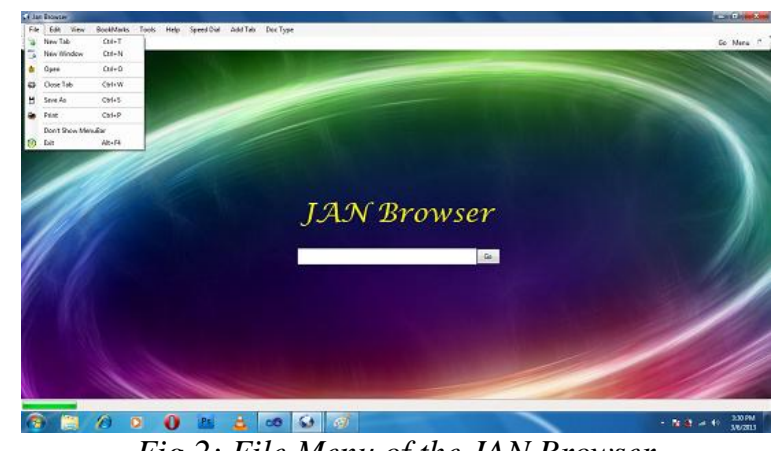

Fig 2: File Menu of the JAN Browser

Using new tab we can open a tab in same window it provide user to view more webpage in same page which is shown in fig 3. 


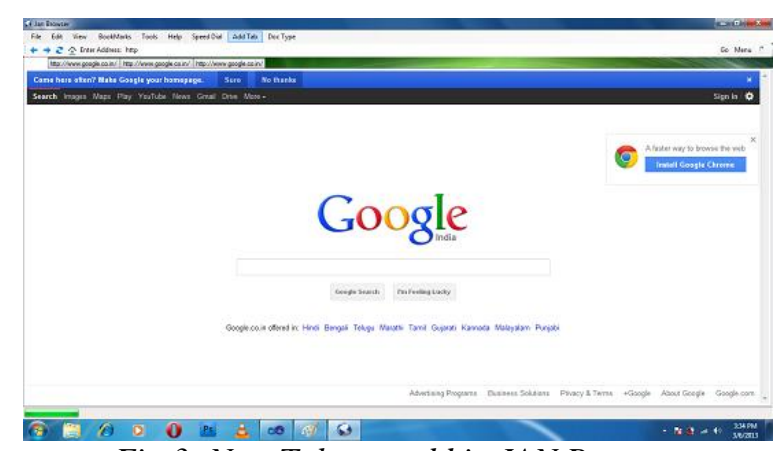

Fig 3: New Tab can add in JAN Browser

The new window option made the user to view the website in separate window it provide speed to network the new window is shown in fig 4.

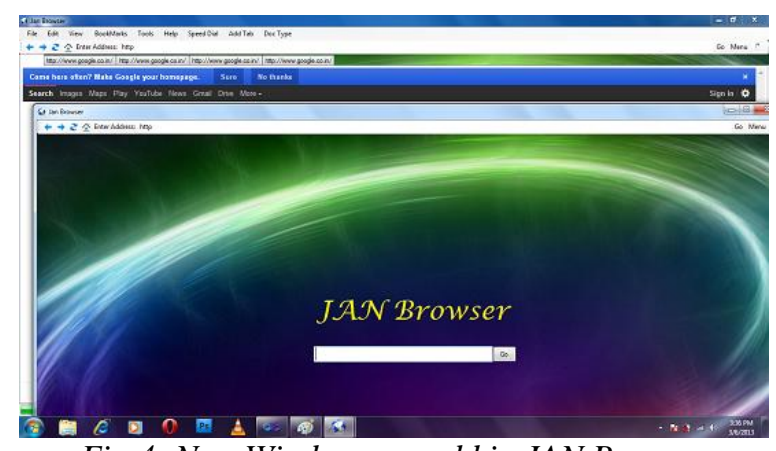

Fig 4: New Window can add in JAN Browser

The next one we discussed is speed dialing; this one is a main feature in JAN browser. It shown in fig 5 this fig describe about the speed dialing for search engine the user can get into search engine through one click.

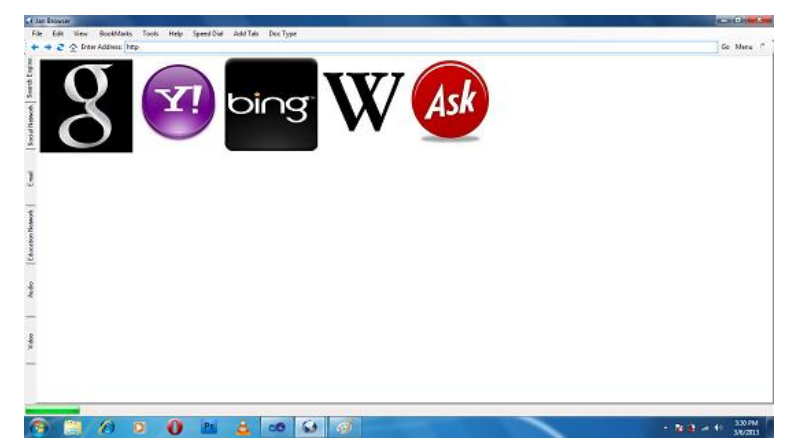

Fig 5: Speed Dialing Tab for search engine in JAN Browser

Example for search engine is shown in fig 6 when we click the $g$ button which shown in fig 5 the result will shown an Google page similarly all button are work as a hyperlink for respective page .

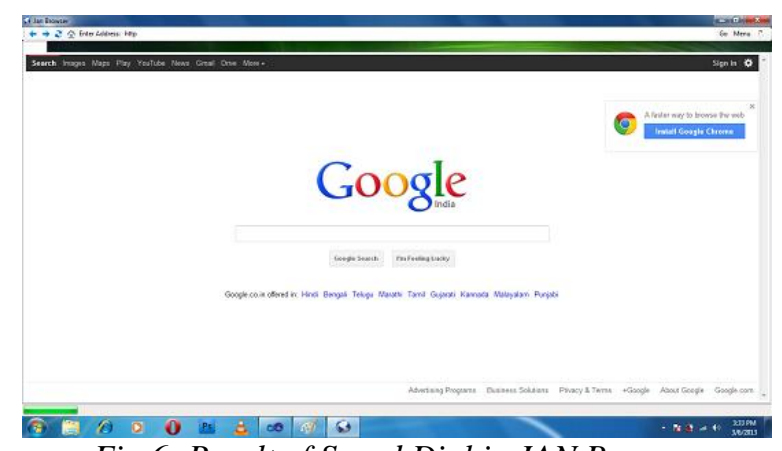

Fig 6: Result of Speed Dial in JAN Browser

Fig 7 represent the another speed dial called Audio, it will show all the audio website the user can enter into the website by click on the button it provide user friendly approach and save the user time 


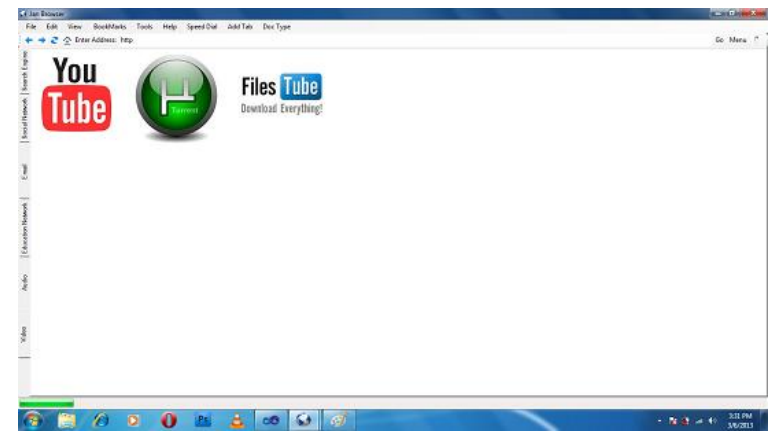

Fig 7: Audio Speed Dial in JAN Browser

The second most special feature is virtual keyboard. This keyboard is had special one called shuffling where each keys are shuffled for each time.

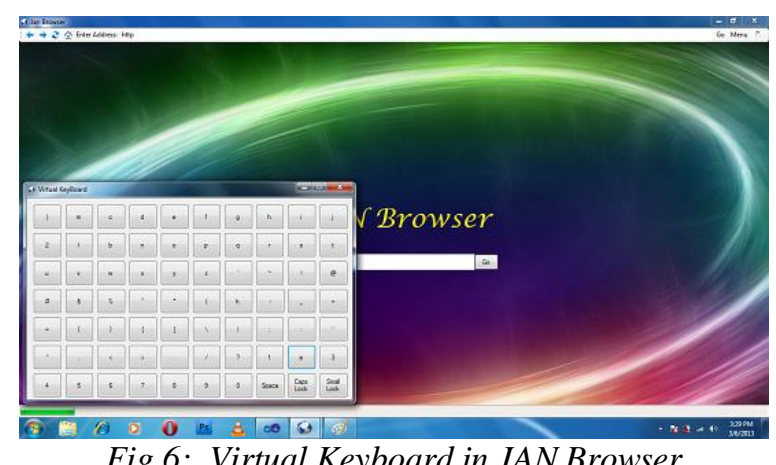

Fig 6: Virtual Keyboard in JAN Browser

Through this method the hacker cant able to hacking the password using screen shot another security also there in normal keyboard when key is pressed the key value is stored in key locker so the hacker can easily hacked it but in virtual keyboard the key value never store in any place so it provide secure to password.

\section{Conclusion}

From this paper, we conclude by saying that, the main motive of developing this JAN browser is to provide all the facilities which make the user to search their needs in browser

- Efficient

- User-friendly

- Secure and

- Save the user valuable time

We use vb.net for developing JAN browser for database purpose we use mysql. JAN browser will satisfied the user need.

\section{Acknowledgements}

We kindly acknowledgement to Ms .M.RAJESWARI ME.,(Ph.D). Assistant Professor, Computer Science and Engineering Department who guided us to make this paper and the application efficiently and successfully.

\section{REFERENCES}

[1] Proposal on a Secure Communication Service Element (SCSE) in the OSI Application Layer, Kouji Nakao and Kenji Suzuki IEEE Journal on selected areas in communications, vol 7, no.4, may 1989.

[2] Performance Models for Automatic Evaluation of Virtual Scanning Keyboards; Samit Bhattacharya, Debasis Samanta and Anupan Basu

IEEE transactions on neural systems and rehabilitation engineering, Vol 16, October 2008

[3] A study of the Energy Consumption Characteristics of Crptographic Algorithms and Security Protocols, Nachiketh R.Pollalpally, Anand Ragunathan IEEE transactions on mobile consumption, vol 5, no 2, February 2006. 